\title{
Phase diagram for one-way traffic flow with local control
}

\author{
Lykov A. A., Malyshev V. A., Melikian M.V. *
}

\begin{abstract}
We consider one-way road deterministic traffic model with $N$ particles. The simplest local control protocol, which reminds physical interaction), with three parameters is considered. We study the stable and unstable domains of the phase diagram üniformly in $N$.
\end{abstract}

\section{Introduction}

Theoretical modelling and computer simulation of transportation systems is a very popular field, see very impressive review [3]. There are two main directions in this research - macro and micro models. Macro approach does not distinguish individual transportation units and uses analogy with the fluid flow in hydrodynamics, see 2]. Stochastic micro models are most popular and use almost all types of stochastic processes: mean field, queueing type and local interaction models. We consider here completely deterministic transportation flows. Although not as popular as stochastic traffic, there is also a big activity in this field, see [4, 5, 6, 7, 8,. In these papers interesting results are obtained for sufficiently general protocols. A popular topics in many papers is to consider optimization of some functional of the chain of cars. For example, in [6, 7] and in references therein, the following model is considered

$$
\ddot{z}_{n}+\alpha \dot{z}_{n}=u_{n}, \quad n=1, \ldots, M,
$$

where $\alpha>0, z_{n}=z_{n}(t)$ is the coordinate of the $n$-th car on the real axis, $u_{1}(t), u_{2}(t), \ldots, u_{M}(t)$ - control functions, which should be chosen to minimize the functional

$$
J=\frac{1}{2} \int_{0}^{\infty} \sum_{n=1}^{M+1} a\left(z_{n-1}(t)-z_{n}(t)-d\right)^{2}+b\left(\dot{z}_{n}(t)-v\right)^{2}+c\left(u_{n}(t)-\alpha v\right)^{2} d t, \quad u_{M+1}=\alpha v .
$$

where $a, b, c$ - some-positive constants, $v, d$ are correspondingly safe velocity and distance between cars. Moreover, it is assumed that

$$
z_{0}(t)=v t, z_{M+1}(t)=v t-(M+1) d,
$$

It is clear however, that any model should satisfy some natural necessary conditions. For example, besides minimizing such functionals, the inequality should hold

$$
I=\inf _{t, n}\left(z_{n-1}(t)-z_{n}(t)\right)>0
$$

that is necessary for safety.

Here we follow another strategy: for simplest possible protocols we try to get results as concrete as possible. Namely, we consider the one-way road traffic model organized as follows.

At any time $t \geq 0$ there is finite or infinite number of point particles (may be called also cars, units etc.) with coordinates $z_{k}(t)$ on the real axis, enumerated as follows

$$
\ldots<z_{n}(t)<\ldots<z_{1}(t)<z_{0}(t)
$$

We assume that the rightmost car (the leader) moves "as it wants", that is the trajectory $z_{0}(t)$ is often assumed to have nonnegative velocity.

Our problem is to find the simplest possible local protocol (control algorithm) which would guarantee both safety (no collisions), stable (or even maximal) density of the flow or maximal current. Otherwise speaking, we try to find control mechanism which guarantees that the distance between any pair of neighbouring cars is close (on all time interval $(0, \infty)$ ) to some (given a priori) fixed number, that defines the density of the flow.

More exactly, denoting $r_{k}(t)=z_{k-1}(t)-z_{k}(t)$, and

${ }^{*}$ Lomonosov Moscow State University, Faculty of Mechanics and Mathematics, Vorobyevy Gory 1, Moscow, 119991, Russia 


$$
I=\inf _{k \geqslant 1} \inf _{t \geqslant 0} r_{k}(t), \quad S=\sup _{k \geqslant 1} \sup _{t \geqslant 0} r_{k}(t),
$$

we try to get the bounds - lower positive bound on $I$ and upper bound on $S$ - as close as possible.

Locality (of the control) means that the "driver" of the $k$-th car, at any time $t$, knows only its own velocity $v_{k}(t)$ and the distance $r_{k}(t)$ from the previous car. Thus, for any $k \geq 1$ the trajectory $z_{k}(t)$, being deterministic, is uniquely defined by the trajectory $z_{k-1}(t)$ of the previous particle.

Using physical terminology one could say that if, for example, $r_{k}(t)$ becomes larger than $d$, then some virtual force $F_{k}$ increases acceleration of the particle $k$, and vice-versa. Thus the control mechanism is of the physical nature, like forces between molecules in crystals but our "forces" are not symmetric. Thus our system is not a hamiltonian system. Nevertheless, our results resemble the dynamical phase transition in the model of the molecular chain rapture under the action of external force, see [9]. However here we do not need the double scaling limit used in [9].

We will see however that for the stability, besides $F_{k}$, also friction force $-\alpha v_{k}(t)$, restraining the growth of the velocity $v_{k}(t)$, is necessary, where the constant $\alpha>0$ should be chosen appropriately. Taking $F_{k}$ to be simplest possible

$$
F_{k}(t)=\omega^{2}\left(z_{k-1}(t)-z_{k}(t)-d\right)
$$

we get that the trajectories are uniquely defined by the system of equations for $k \geq 1$

$$
\frac{d^{2} z_{k}}{d t^{2}}=F_{k}(t)-\alpha \frac{d z_{k}}{d t}=\omega^{2}\left(z_{k-1}(t)-z_{k}(t)-d\right)-\alpha \frac{d z_{k}}{d t}
$$

Stability depends not only on the parameters $\alpha, \omega, d$ but also on the initial conditions and on the movement of the leader (on its velocity and acceleration). This is easy to understand for the case of $N+1$ particles. For example, for $N=1$, where the calculations are completely trivial, assume also the simplest leader movement

$$
z_{0}(t)=v t, t \geq 0
$$

Then, if initial condition for the second particle are

$$
z_{1}(0)=-a=-\left(d+\frac{\alpha}{\omega^{2}} v\right), \dot{z}_{1}(0)=v,
$$

then $z_{1}(t)=-a+v t$ for any $d, \alpha, \omega$. However, if we change only the initial velocity $\dot{z}_{1}(0)=w$ to some $w>0$, then for any $\alpha, \omega$ there exists $w_{1}=w_{1}(\alpha, \omega, d)$ such that for any $w \geq w_{1}$ collision occurs.

For $N=2,3, \ldots$ the situation becomes more and more complicated, and its study has no much sense. That is why we study, in the space of two parameters $\alpha, \omega$ (for fixed $d$ ), stability conditions, which are uniform in $N$ and in large class of reasonable initial conditions and reasonable movement of the leader.

Natural (reasonable) initial conditions are as follows: at time 0 it should be

$$
0<\inf _{k \geqslant 1} r_{k}(0) \leq \sup _{k \geqslant 1} r_{k}(0)<\infty
$$

As for the leader movement, it is sometimes sufficient to assume that the function $z_{0}(t)$ were continuous, but in other cases it is assumed to twice differentiable and has the following bounds on the velocity and acceleration of the leader:

$$
\sup _{t \geqslant 0}\left|\dot{z}_{0}(t)\right|=v_{\max }, \quad \sup _{t \geqslant 0}\left|\ddot{z}_{0}(t)\right|=a_{\max },
$$

It appears that under these conditions there are 3 sectors in the quarter-plane $\left.R_{+}^{2}=\{(\alpha, \omega)\}: 1\right) \alpha>2 \omega$, where we can prove stability, 2) $\alpha<\sqrt{2} \omega$, where we can prove instability, and the sector 3) $\sqrt{2} \omega \leq \alpha \leq 2 \omega$, where we can prove stability only for more restricted classes of initial conditions and of the leader motion.

\section{Results}

\subsection{Stability}

Here we consider the region $\alpha>2 \omega$, and this is always assumed in this section. 
Movement close to stationary For any given $d>0$ there are special initial conditions (which can be called equilibrium configuration) when the force acting on any particle is zero:

$$
z_{k}(0)=-k a, \dot{z}_{k}(0)=v, k=0,1,2, \ldots,
$$

with

$$
a=a(\alpha, \omega, d, v)=d+\frac{\alpha}{\omega^{2}} v
$$

If the leader moves as (4), then for any $k>0$ also

$$
z_{k}(t)=z_{k}(0)+v t
$$

Such movement we call stationary. Now consider a perturbation of this situation

Theorem 1 For given $d$ and $v$ assume

$$
\begin{gathered}
\left|z_{k}(0)+k a\right| \leqslant \theta a, \quad\left|\dot{z}_{k}(0)-v\right| \leqslant \beta v \\
\sup _{t \geqslant 0}\left|z_{0}(t)-v t\right| \leqslant \delta a
\end{gathered}
$$

for $0 \leq \delta<\frac{1}{2}$ and $\theta, \beta \geqslant 0$ such that

$$
\epsilon=2 \max \left\{\delta, \frac{\theta+\frac{2 v \beta}{\alpha a}}{\sqrt{1-\left(\frac{2 \omega}{\alpha}\right)^{2}}}\right\}<1
$$

Then

$$
(1-\epsilon) a \leqslant I \leqslant S \leqslant(1+\epsilon) a
$$

Now we want to show that in some cases the sufficient conditions of this theorem are are not very far from necessary. For example, from the theorem follows that the condition

$$
\theta<\frac{1}{2} \sqrt{1-\left(\frac{2 \omega}{\alpha}\right)^{2}}, \delta=\beta=0
$$

is sufficient for $I>0$. This means that the deviations of the particle from the equilibrium (that is the distances between particles are equal to $a$ ) do not exceed $\frac{a}{2}$.

Remark 1 The movement of any first $N$ particles does not depend on other particles. Thus for any $N$ the system of equations (3) for $k=1, \ldots, N$, is finite-dimensional, linear and, for any $\alpha, \omega>0$, the spectrum of this linear operator is inside the left half-plane. One could look at it formally, neglecting possible collisions between particles. In this case it is asymptotically stationary, that is, as $t \rightarrow \infty$, its solution converges to stationary for any initial conditions and if $z_{0}(t)=v t$. However we do not know whether collisions occur before it becomes stationary. In the following theorem we get such conditions.

Because of this one could think that the spectrum does not play big role in the stability problems. This is not quite true if we consider the problems uniform in $N$, or the corresponding infinite-dimentional operator, see below.

Note also that for the random movement of the leader the same results hold. More exactly, assume that $z_{0}(t)$ is a stationary process with smooth trajectories, satisfying bounds (5) with probability one. Then the mean velocity of any particle converges to the mean velocity of the leader.

Theorem 2 1) Assume (6) and (9). Then for all $k=1,2, \ldots$ we have:

$\sup _{t \geqslant 0}\left|\dot{z}_{k}(t)-v\right| \leqslant \sup _{t \geqslant 0}\left|\dot{z}_{0}(t)-v\right|$. If the righthand side is sufficiently small then $v_{k}(t)=\dot{z}_{k}(0)>0$ for any $k, t$.

2) Assume (4), (8) and that for some parameters $\theta, \beta>0$

$$
\zeta=2 \frac{\theta+\frac{2 v \beta}{\alpha a}}{\sqrt{1-\left(\frac{2 \omega}{\alpha}\right)^{2}}}<1
$$

Then for all $k$

$$
\lim _{t \rightarrow \infty}\left(z_{k}(t)-(v t-k a)\right)=0
$$

where $a$ is in (7) and

$$
(1-\zeta) a \leqslant I \leqslant S \leqslant(1+\zeta) a .
$$


Non-stationary initial conditions In Theorems (1) and (2) we considered initial coordinates of the particles close to the fixed lattice points $-k a$. Here we consider more natural initial conditions with restrictions only on the distances between particles. Denote

$$
d^{*}=\frac{a_{\max }+\alpha v_{\max }}{\omega^{2}}
$$

Theorem 3 Let the initial conditions be

$$
(1-\theta) d \leqslant r_{k}(0) \leqslant(1+\theta) d, \quad\left|\dot{z}_{k-1}(0)-\dot{z}_{k}(0)\right| \leqslant \beta, \quad k=1,2, \ldots
$$

for some $\beta \geqslant 0,0 \leqslant \theta<1$. Assume moreover that

$$
\eta=\max \left(\frac{d^{*}}{d}, \frac{\theta+\frac{2 \beta}{\alpha d}}{\sqrt{1-\left(\frac{2 \omega}{\alpha}\right)^{2}}}\right)<1
$$

Then we have the following stability bounds

$$
(1-\eta) d \leqslant I \leqslant S \leqslant(1+\eta) d
$$

Remark 2 If $\beta=\theta=0$, one can prove the same result for the case $\alpha=2 \omega$.

Corollary 1 Assume that

$$
r_{k}(0)=z_{k-1}(0)-z_{k}(0)=d, \quad \dot{z}_{k}(0)=\dot{z}_{0}(0), \quad k=1,2, \ldots
$$

If moreover

$$
d^{*}<d
$$

then

$$
d-d^{*} \leqslant I \leqslant S \leqslant d+d^{*}
$$

Remark 3 It is natural to consider the stability problem for more general initial conditions. Assume we know the initial conditions and the parameters $v_{\max }, a_{\max }$ of the leader. We want to know whether the parameters $\alpha, \omega, d$ such that $0<I \leqslant S<\infty$, exist. Namely, we will prove that for any $z_{0}(t)$, satisfying (5) and the initial conditions such that:

$$
\min _{k \geqslant 1}\left(z_{k-1}(0)-z_{k}(0)\right)=A>0, \quad \max _{k \geqslant 1}\left(z_{k-1}(0)-z_{k}(0)\right)=B<\infty, \quad \max _{k \geqslant 1}\left|\dot{z}_{k-1}(0)-\dot{z}_{k}(0)\right|=C<\infty,
$$

the parameters $\alpha, \omega, d$, such that $0<I \leqslant S<\infty$, always exist.

Theorem 1, 3 and Corollary 1

1) guarantee that there are no collisions between particles (by the lower bound),

2) provide lower bound for the density of the flow (by the upper bound), that is the mean distance between particles remains bounded.

3) do not guarantee that the velocities remain positive. Such conditions were obtained above in Theorem 2 ,

Flow density Let $n(t, I)$ be the number of units on the interval $I \subset R$ at time $t$. Instead of the density $(|I|$ is the length of $I$ )

$$
\rho(t)=\liminf _{|I| \rightarrow \infty} \frac{n(t, I)}{|I|}
$$

it is more convenient to consider the inverse density, or the mean length of the chain of cars $0,1, \ldots, N$

$$
L_{N}(t)=\frac{z_{0}(t)-z_{N}(t)}{N} .
$$

Theorem 4 For any $\alpha>0, \omega>0$ assume that the initial conditions (15) are such that the following finite limits exist

Then for any $t$ there exists

$$
\lim _{N \rightarrow \infty} L_{N}(0)=L(0), \quad \lim _{N \rightarrow \infty} \dot{L}_{N}(0)=\dot{L}(0),
$$

$$
\lim _{N \rightarrow \infty} L_{N}(t)=L(t)
$$

and moreover

$$
L(t)=L(0)+\frac{1}{\alpha}\left(1-e^{-\alpha t}\right) \dot{L}(0)
$$


Note that if moreover $\frac{d z_{k}(0)}{d t}$ are uniformly bounded then $\frac{d L}{d t}(0)=0$, that is the mean length does not change with time.

Due to convergence to stationary movement, the flow current converges, at any point, to the current of the stationary flow

$$
v a^{-1}=\frac{\omega^{2} v}{\omega^{2} d+\alpha v}
$$

Restricted stability Here we consider the region $\sqrt{2} \omega \leq \alpha \leq 2 \omega$, where we can prove stability only for asymptotically homogeneous initial conditions.

Theorem 5 Let $\sqrt{2} \omega \leqslant \alpha \leq 2 \omega$ and let $z_{0}(t)$ be such that

$$
\omega \int_{0}^{\infty}\left|z_{0}(t)-v t\right| d t=\sigma a<\infty
$$

for some $\sigma \geqslant 0$. Assume also that the initial conditions are "summable", that is

$$
\sum_{k=1}^{\infty}\left|z_{k}(0)+k a\right| \leqslant \theta a, \quad \sum_{k=1}^{\infty}\left|\dot{z}_{k}(0)-v\right| \leqslant \beta v
$$

for some $\theta, \beta \geqslant 0$. Then

$$
I \geqslant(1-2 \eta) a, \quad S \leqslant(1+2 \eta) a,
$$

where

$$
\eta=2\left(\theta+\frac{\beta v}{a \omega}+\sigma\right)
$$

It follows from this theorem that the upper bound $S<\infty$ holds for all parameters, but the lower (safety) bound $I>0$ holds if

$$
\theta+\frac{\beta v}{a \omega}+\sigma<\frac{1}{4}
$$

Theorem 6 Assume again that $\sqrt{2} \omega \leqslant \alpha \leq 2 \omega$, the initial conditions satisfy

$$
\sum_{k=1}^{\infty}\left|r_{k}(0)-d\right| \leqslant \theta d, \quad \sum_{k=1}^{\infty}\left|\dot{z}_{k-1}(0)-\dot{z}_{k}(0)\right| \leqslant \beta, \quad k=1,2, \ldots
$$

for some $\beta \geqslant 0,0 \leqslant \theta \leqslant 1$, and the leader moves as

$$
\frac{1}{\omega} \int_{0}^{+\infty}\left|\ddot{z}(t)+\alpha \dot{z}_{0}(t)\right| d t=\sigma d<\infty
$$

for some $\sigma \geqslant 0$. Then

$$
I>d(1-\eta), \quad S<d(1+\eta),
$$

where

$$
\eta=2\left(\theta+\frac{\beta}{\omega d}+\sigma\right), \quad x=\frac{\alpha}{\omega} .
$$

It follows that the safety condition $I>0$ holds if

$$
\theta+\frac{\beta}{\omega d}+\sigma<\frac{1}{2}
$$




\subsection{Instability}

Here we will prove instability for the region $\alpha<\sqrt{2} \omega$. The first reason for the instability is the absence of dissipation, that is if $\alpha=0$. The following result shows this even for the most favorable initial conditions.

Theorem 7 Assume that $\alpha=0$ and

$$
\begin{gathered}
z_{k}(0)=-k a, \frac{d z_{k}}{d t}(0)=v, k \geq 0 \\
z_{0}(t)=t v+\sin \omega_{0} t, v>0, \omega_{0} \neq 0
\end{gathered}
$$

Then for any $k \geqslant 2$ we have due to resonance

$$
\inf _{t \geqslant 0} r_{k}(t)=-\infty
$$

Now we show that, even under the smallest perturbation of the initial conditions (19) and even simpler leader trajectory, we get much more general instability condition.

Theorem 8 Assume $\alpha<2 \omega, z_{0}(t)=v t$ and initial conditions such that:

$$
z_{k}(0)=-k a, \dot{z}_{k}(0)=\left\{\begin{array}{ll}
v, & k>1 \\
v+\epsilon, & k=1
\end{array},\right.
$$

where $\epsilon$ is some real number. Then for any $\mu>\frac{1}{\tau}, \tau=\sqrt{\omega^{2}-\frac{\alpha^{2}}{4}}$ the following asymptotic formula takes place:

$$
z_{k+1}(t)-(v t-(k+1) a) \sim \frac{c}{\sqrt{k}} e^{k f(\mu)} \sin \left(\Omega(\mu) k+\phi_{0}(\mu)\right), \text { when } t=\mu k, k \rightarrow \infty
$$

where

$$
\begin{gathered}
f(\mu)=-\frac{\alpha \mu}{2}+1-\ln \left(\frac{2 \tau}{\mu \omega^{2}}\right), \\
\phi_{0}(\mu)=\arctan (\nu), \nu=\sqrt{\mu^{2} \tau^{2}-1}, \Omega(\mu)=\nu-\phi_{0}(\mu)=\nu-\arctan (\nu), c=\epsilon \sqrt{\frac{2 \tau}{\pi \nu \mu}}
\end{gathered}
$$

Corollary 2 Assume $\alpha<\sqrt{2} \omega$ and $z_{0}(t)=v t$. Assume the initial conditions (20). Then

$$
I=-\infty, \quad S=\infty .
$$

While proving the theorem we will see that corollary (2) holds even for more general initial conditions:

$$
\max _{k}\left|z_{k}(0)+k a\right| \leqslant \epsilon_{1}, \quad \max _{k}\left|\dot{z_{k}}(0)-v\right| \leqslant \epsilon_{2} .
$$

with some nonnegative $\epsilon_{1}, \epsilon_{2} \geqslant 0$.

\section{Stability: proofs}

\subsection{Theorem [3, Corollary 1, Remark 1}

It is very convenient to use new variables

$$
x_{0}(t)=\frac{\ddot{z}_{0}(t)+\alpha \dot{z}_{0}(t)}{\omega^{2}}, x_{k}(t)=z_{k-1}(t)-z_{k}(t)-d=r_{k}(t)-d, \quad k=1,2, \ldots
$$

Using

$$
\ddot{z}_{k}=\omega^{2} x_{k}-\alpha \dot{z}_{k}=\omega^{2} x_{k}-\alpha\left(\dot{z}_{k-1}-\dot{x}_{k}\right), \ddot{z}_{k}=\ddot{z}_{k-1}-\ddot{x}_{k}
$$

we get the main equations (3) in terms of these variables:

$$
\ddot{x}_{k}+\alpha \dot{x}_{k}+\omega^{2} x_{k}=\ddot{z}_{k-1}+\alpha \dot{z}_{k-1}=\omega^{2} x_{k-1}, \quad x_{0}=\frac{\ddot{z}_{0}+\alpha \dot{z}_{0}}{\omega^{2}} .
$$


Lemma 1 Assume that

$$
\sup _{t \geqslant 0}\left|x_{0}(t)\right|=Q<\infty
$$

Let also the initial conditions be such that

$$
\sup _{k \geqslant 1}\left|x_{k}(0)\right|=A<\infty, \quad \sup _{k \geqslant 1}\left|\dot{x}_{k}(0)\right|=C<\infty .
$$

If $\alpha>2 \omega$ then

$$
\sup _{k, t}\left|x_{k}(t)\right| \leqslant Q^{\prime}=\max \left\{Q, \frac{\alpha A+2 C}{\sqrt{\alpha^{2}-4 \omega^{2}}}\right\} .
$$

Proof. The solution of the linear equation (22) for any fixed $k$ is of course well-known. Namely, if the roots

$$
\lambda_{ \pm}=-\frac{\alpha}{2} \pm \sqrt{\frac{\alpha^{2}}{4}-\omega^{2}}
$$

of the characteristic equation

$$
G(\lambda)=\lambda^{2}+\alpha \lambda+\omega^{2}=0
$$

are different, then the solution can be written as

$$
x_{k}(t)=x_{k,+}(t)+x_{k,-}(t)
$$

where

$$
\begin{gathered}
x_{k, \pm}(t)=C_{\mathrm{K}, \pm} e^{\lambda_{ \pm} t}+\omega^{2} \frac{e^{\lambda_{ \pm} t}}{Q^{\prime}\left(\lambda_{ \pm}\right)} \int_{0}^{t} e^{-\lambda_{ \pm} t_{1}} x_{k-1}\left(t_{1}\right) d t_{1}= \\
=C_{\mathrm{K}, \pm} e^{\lambda_{ \pm} t}+\omega^{2} \frac{e^{\lambda_{ \pm} t}}{2 \lambda_{ \pm}+\alpha} \int_{0}^{t} e^{-\lambda_{ \pm} t_{1}} x_{k-1}\left(t_{1}\right) d t_{1}
\end{gathered}
$$

Using the initial conditions one finds

$$
C_{k, \pm}=\frac{1}{2 \gamma}\left(\left( \pm \frac{\alpha}{2}+\gamma\right) a_{k} \pm b_{k}\right), \gamma=\sqrt{\frac{\alpha^{2}}{4}-\omega^{2}}
$$

where $x_{k}(0)=a_{k}, k \in \mathbb{N}, \dot{x}_{k}(0)=b_{k}, k \in \mathbb{N}$. As $\lambda_{+}>\lambda_{-}$we get:

$$
\left|x_{k}(t)\right| \leq\left|C_{\mathrm{K},+}\right| \exp \left(\lambda_{+} t\right)+\left|C_{k,-}\right| \exp \left(\lambda_{-} t\right)+\sup _{s \geq 0}\left|x_{k-1}(s)\right| \frac{\omega^{2}}{2 \gamma} \int_{0}^{t}\left|\exp \left(\lambda_{+}\left(t-t_{1}\right)\right)-\exp \left(\lambda_{-}\left(t-t_{1}\right)\right)\right| d t_{1}
$$

Let us prove that for $y_{k}=\sup _{s \geq 0}\left|x_{k}(s)\right|$ the following inequalities hold

$$
y_{k} \leq \max \left\{y_{k-1}, \frac{\alpha A+2 C}{2 \gamma}\right\} .
$$

Putting $t-t_{1}=s$ and using $\omega^{2}=\lambda_{+} \lambda_{-}$and

$$
\left|C_{k, \pm}\right| \leq \frac{\left(\frac{\alpha}{2} \pm \gamma\right) A+C}{2 \gamma}
$$

we get:

$$
\begin{gathered}
\left|x_{k}(t)\right| \leq\left|C_{\mathrm{\kappa},+}\right| \exp \left(\lambda_{+} t\right)+\left|C_{k,-}\right| \exp \left(\lambda_{-} t\right)+\sup _{s \geq 0}\left|x_{k-1}(s)\right| \frac{\omega^{2}}{2 \gamma} \int_{0}^{t}\left(\exp \left(\lambda_{+} s\right)-\exp \left(\lambda_{-} s\right)\right) d s \leq \\
\leq \frac{1}{2 \gamma}\left(\left(\frac{\alpha}{2}+\gamma\right) A+C+y_{k-1} \lambda_{-}\right) \exp \left(\lambda_{+} t\right)+\frac{1}{2 \gamma}\left(\left(\frac{\alpha}{2}-\gamma\right) A+C-y_{k-1} \lambda_{+}\right) \exp \left(\lambda_{-} t\right)+y_{k-1}
\end{gathered}
$$

To finish the proof we need the following simple lemma.

Lemma 2 Let us consider the function

$$
f(t)=a \exp \left(\lambda_{+} t\right)+b \exp \left(\lambda_{-} t\right)+c
$$

for some constants $b, c>0, a \in \mathbb{R}, \lambda_{-}<\lambda_{+}<0$. For every $t \geq 0$ the following statement is true:

$$
|f(t)| \leq \max \{c, a+b+c\} .
$$


Proof. There are two cases:

1. $a>0$, then it is obvious that $\sup _{s \geq 0}|f(s)|=f(0)=a+b+c$.

2. $a<0$, in this case the set of such $\bar{t}$, that the derivative of the function is positive 0 , is:

$$
t>\frac{1}{\lambda_{-}-\lambda_{+}} \ln \left(-\frac{a}{b} \frac{\lambda_{+}}{\lambda_{-}}\right)
$$

The point $t_{0}=\frac{1}{\lambda_{-}-\lambda_{+}} \ln \left(-\frac{a}{b} \frac{\lambda_{+}}{\lambda_{-}}\right)$is the minimum point. If $t_{0}<0$, then $\sup _{s \geq 0}|f(s)|$ tops in the point $+\infty$ and is equal to $c$. If $t_{0}>0$, then $\sup _{s>0}|f(s)|$ tops in the point 0 or in the point $+\infty$.

Having applied this lemma to (25) we get (24) and

$$
\left|x_{k}(t)\right| \leq \max \{Q, B\}=Q^{\prime}
$$

where $B=\frac{\alpha A+2 C}{2 \gamma}$. Lemma 1 is thus proved.

To prove theorem 3 it is sufficient to put $A=\theta d, C=\beta, Q=d^{*}$. To prove corollary 1 we can put $\theta=\beta=0$.

Remark We use here theorem 3 and the notation therein. From

$$
(1-\theta) d=A, \quad(1+\theta) d=B, \quad \beta=C,
$$

we find first the parameters $d, \theta$,

$$
d=\frac{A+B}{2}, \quad \theta=\frac{B-A}{2 d}=\frac{B-A}{A+B}, .
$$

Then we find $\alpha, \omega$ such that $\alpha>2 \omega$ and $\eta<1$. Namely, we choose $\alpha$ sufficiently large and take any $\omega$ so that

$$
\frac{1}{\sqrt{d}} \sqrt{a_{\max }+\alpha v_{\max }}<\omega<\frac{\alpha}{2}
$$

Then the inequality (16) evidently holds.

\subsection{Theorem 1}

Note that the functions $q_{k}(t), k=1,2 \ldots$, defined by

$$
z_{k}(t)=v t-k a+q_{k}(t),
$$

satisfy the system of equations (22) with the initial conditions:

$$
q_{0}(t)=z_{0}(t)-v t, \quad q_{k}(0)=z_{k}(0)+k a, \quad \dot{q}_{k}(0)=\dot{z}_{k}(0)-v, \quad k=1,2 \ldots
$$

Then

$$
\sup _{k}\left|q_{k}(0)\right| \leqslant \theta a, \quad \sup _{k}\left|\dot{q}_{k}(0)\right| \leqslant \beta v, \quad \sup _{t}\left|q_{0}(t)\right|=\delta a .
$$

Using lemma 1 one has the lower bound

$$
r_{k}(t)=z_{k-1}(t)-z_{k}(t)=a+q_{k-1}-q_{k}(t) \geqslant a-2 \sup _{k, t}\left|q_{k}(t)\right|=a(1-\epsilon) .
$$

The upper bound for $r_{k}(t)$ can be obtained similarly.

\subsection{Theorem 2}

Differentiating the system (22) we get the equations for velocities $v_{k}(t)=\dot{z}_{k}(t)$.

$$
\ddot{v}_{k}=\omega^{2}\left(v_{k-1}-v_{k}\right)-\alpha \dot{v}_{k},
$$

with initial conditions:

$$
v_{k}(0)=v, \dot{v}_{k}(0)=0
$$

So for every $k=0,1,2, \ldots$ :

$$
v_{k}(t)=v+u_{k}(t),
$$


where $u_{k}$ satisfy equations (28) with zero initial conditions $u_{0}(t)=v_{0}(t)-v, u_{k}(0)=\dot{u}_{k}(0)=0, k \geq 1$. So from lemma :1 for $u_{k}$ we get that for any $k=1,2, \ldots$ and $t \geqslant 0$

$$
\left|u_{k}(t)\right| \leqslant \sup _{t \geqslant 0}\left|u_{0}(t)\right|=\sup _{t \geqslant 0}\left|\dot{z}_{0}(t)-v\right|
$$

This proves assertion 1) of the Theorem.

Let us prove now assertion 2). The functions $q_{k}(t)$ defined in (27) satisfy the system (22) with initial conditions:

$$
q_{k}(0)=z_{k}(0)+k a, \quad \dot{q}_{k}(0)=\dot{z}_{k}(0)-v .
$$

Moreover $q_{0}(t)=z_{0}(t)-v t=0$ for all $t \geqslant 0$. By induction we get:

$$
\left(\frac{d^{2}}{d t^{2}}+\alpha \frac{d}{d t}+\omega^{2}\right)^{k} q_{k}=0
$$

which gives

$$
q_{k}(t)=e^{\lambda_{1} t} P_{k}(t)+e^{\lambda_{2} t} Q_{k}(t)
$$

where $P_{k}, Q_{k}$ are the polynomials of degree $k-1$ and $\lambda_{1}, \lambda_{2}$ are the roots of the equation (23). Note that, for any $\omega, \alpha>0$, the real parts of $\lambda_{1}, \lambda_{2}$ are negative.

The last statement follows if we put $\delta=0$ in (10), which gives (13).

\subsection{Theorems 5 and 6}

Before proving them, we need new notation. Introduce the Banach space

$$
X=\left\{\psi=(q, p), q=\left\{q_{k}\right\}_{k=1,2 \ldots}, p=\left\{p_{k}\right\}_{k=1,2 \ldots}:\|q\|=\sup _{k}\left|q_{k}\right|<\infty,\|p\|=\sup _{k}\left|p_{k}\right|<\infty, \quad q_{k}, p_{k} \in \mathbb{R}\right\}
$$

with the norm

$$
\|\psi\|=\max \{\|q\|,\|p\|\} .
$$

Define also the bounded (non-selfadjoint) linear operator in $X$

$$
A \psi=\psi^{\prime}, \quad \psi=(q, p), \psi^{\prime}=\left(q^{\prime}, p^{\prime}\right),
$$

where

$$
q^{\prime}=p, \quad p_{k}^{\prime}=\omega^{2}\left(q_{k-1}-q_{k}\right)-\alpha p_{k}, \quad q_{0}=0
$$

Then the dynamics (22), if $q_{k}=x_{k}, p_{k}=\dot{x}_{k}, k \geq 1$ and $x_{0}(t)=0$, can be written as

$$
\dot{\psi}=A \psi \Longrightarrow \psi(t)=e^{A t} \psi(0)
$$

Proof of theorem 5 and 6 is similar to the proof of theorems 1 and 3 , but one should use, instead of lemma 1. the following lemma.

Lemma 3 Consider the system of equations

$$
\frac{d^{2} q_{k}}{d t^{2}}+\alpha \frac{d q}{d t}+\omega^{2} q_{k}=\omega^{2} q_{k-1}, k=1,2, \ldots
$$

that is the same as (22), where $q_{0}(t)$ is continuous and absolutely integrable on $\mathbb{R}_{+}$:

$$
\int_{0}^{+\infty}\left|q_{0}(t)\right| d t=Q<\infty
$$

Assume the following initial conditions

$$
\sum_{k=1}^{\infty}\left|q_{k}(0)\right|=a<\infty, \quad \sum_{k=1}^{\infty}\left|\dot{q}_{k}(0)\right|=b<\infty .
$$

If $\sqrt{2} \leqslant \alpha \leq 2 \omega$ then

$$
\sup _{k, t}\left|q_{k}(t)\right| \leqslant Q^{\prime}=2 a+\frac{2 b}{\omega}+2 \omega Q .
$$


Proof. The system (32) can be written in the operator form, as in

$$
\dot{\psi}=A \psi+\omega^{2} q_{0}(t) g_{1}, \psi(0)=\psi,
$$

where $g_{1}=\left(0, e_{1}\right)^{T} \in X, e_{1} \in$ is the vector $(1,0,0,0 \ldots)$. Then the solution of (33) is quite standard, see [10],

$$
\psi(t)=e^{t A} \psi(0)+\omega^{2} \int_{0}^{t} q_{0}(s) e^{(t-s) A} g_{1} d s .
$$

and

$$
e^{t A}=-\frac{1}{2 \pi i} \int_{\Gamma} e^{z t} R(z) d z,
$$

where $\Gamma$ is a closed contour around the spectrum of $A$. Using lemma 5 we get

$$
q_{k}(t)=q_{k}^{(L)}(t)+q_{k}^{(N)}(t),
$$

where

$$
\begin{aligned}
& q_{k}^{(L)}(t)=\left(e^{t A} \psi(0)\right)\left(q_{k}\right)=-\frac{1}{2 \pi i} \sum_{j=0}^{k-1} \int_{\Gamma} \phi^{j}(z) l_{k-j}(z) e^{t z} d z \\
& q_{k}^{(N)}(t)=\frac{\omega^{2}}{2 \pi i} \int_{\Gamma} \frac{\phi^{k-1}(z)}{z^{2}+\alpha z+\omega^{2}} Q(t, z) d z, \quad Q(t, z)=\int_{0}^{t} e^{(t-s) z} q_{0}(s) d s .
\end{aligned}
$$

Then change of variables gives

$$
Q(t, z)=\int_{0}^{t} e^{s z} q_{0}(t-s) d s=\sum_{k=0}^{\infty} \frac{z^{k}}{k !} \int_{0}^{t} s^{k} q_{0}(t-s) d s .
$$

As for any $t \geqslant 0$

$$
\left|\int_{0}^{t} s^{k} q_{0}(t-s) d s\right| \leqslant t^{k} Q,
$$

the $Q(t, z)$ is entire for any $t \geqslant 0$. Note that the integrands in the formulas (36)-(37) are meromorphic functions in the complex plane, having two exactly two poles $z_{1}, z_{2}$, corresponding to the multiplicity of the roots of the polynomial $G(z)$. By theorem 9 the points $z_{1}, z_{2}$ belong to the spectrum of $A$, and by the same theorem, we can to choose the contour $\Gamma$ so that the following 3 conditions hold

1. if $z \in \Gamma$ then $\operatorname{Re}(z) \leqslant 0$;

2. $\sigma(A) \cap \Gamma=\{0\}$;

3. the points $z_{1}, z_{2}$ lie inside the domain bounded by $\Gamma$.

Using formulas (36)-(37) for the chosen $\Gamma$, we will get estimates of the functions $q_{k}^{(L)}, q_{k}^{(N)}$. It is clear that $|\phi(z)| \leqslant 1$ for all $z \in \Gamma$. Then

$$
\left|q_{k}^{(L)}(t)\right| \leqslant \frac{1}{2 \pi} \sum_{j=0}^{k-1} \int_{\Gamma}\left|\phi^{j}(z)\right|\left|l_{k-j}(z)\right|\left|e^{t z}\right||d z| \leqslant c_{q}^{\prime} a+c_{p}^{\prime} b,
$$

where the constants

$$
c_{q}^{\prime}=\frac{|\Gamma|}{2 \pi} \frac{1}{\omega^{2}} \max _{z \in \Gamma}|\alpha+z|, \quad c_{p}^{\prime}=\frac{|\Gamma|}{2 \pi} \frac{1}{\omega^{2}} .
$$

Similarly we get estimate for $q_{k}^{(N)}$ :

$$
\left|q_{k}^{(N)}(t)\right| \leqslant Q \frac{|\Gamma|}{2 \pi} .
$$

We want to estimate the length of the contour $\Gamma$, satisfying the conditions 1-3. Assuming that $\alpha<2 \omega$, let the points $z_{1}, z_{2}$ are enumerated so that

$$
z_{1}=-\frac{\alpha}{2}+i r, \quad z_{2}=-\frac{\alpha}{2}-i r, \quad r=\sqrt{\omega^{2}-\frac{\alpha^{2}}{4}} .
$$


Then $z^{2}+\alpha z+\omega^{2}=\left(z-z_{1}\right)\left(z-z_{2}\right)$, and if $z \in \sigma(A)$, then at least one of the inequalities $\left|z-z_{1}\right| \leqslant \omega$ or $\left|z-z_{2}\right| \leqslant \omega$ holds, see Theorm 9 below. It follows that $\sigma(A)$ belongs to the union of two circles

$$
K_{1}=\left\{z \in \mathbb{C}:\left|z-z_{1}\right| \leqslant \omega\right\}, \quad K_{2}=\left\{z \in \mathbb{C}:\left|z-z_{1}\right| \leqslant \omega\right\} .
$$

Note that both circles intersect the real axis at the points $-\alpha$ and 0 . That is why $\Gamma$ can be presented as the union of a part of the circle bounding $K_{1}$, segment of the imaginary axis and a part of the circle bounding $K_{2}$. Then

$$
|\Gamma| \leqslant 4 \pi \omega, \quad \max _{z \in \Gamma}|z+\alpha|=2 \omega .
$$

and finally

$$
\left|q_{k}^{(L)}(t)\right| \leqslant 2 a+\frac{2 b}{\omega}, \quad\left|q_{k}^{(N)}(t)\right| \leqslant 2 \omega Q
$$

The proof of Theorem [6 is similar to the proof of Theorem [5] only instead of the variables $q_{k}(t)$, introduced therein, one should use variables $x_{k}(t)$, introduced in (21).

\subsection{Theorem 4}

Rewriting $L_{N}$ in terms of $x_{k}$

$$
L_{N}(t)=\frac{z_{0}(t)-z_{N}(t)}{N}=\frac{\sum_{k=1}^{N} x_{k}(t)}{N}+d, t \geqslant 0
$$

and using the equations (22), we get the linear equation for $L_{N}$

$$
\ddot{L}_{N}+\alpha \dot{L}_{N}+\omega^{2} L_{N}=\frac{\omega^{2}}{N} \sum_{k=1}^{N} x_{k-1}+d \omega^{2}=\omega^{2}\left(\sum_{k=1}^{N} \frac{x_{k}}{N}+d+\frac{x_{0}-x_{N}}{N}\right)=\omega^{2} L_{N}+\omega^{2} \frac{x_{0}-x_{N}}{N}
$$

or

$$
\ddot{L}_{N}+\alpha \dot{L}_{N}=f_{N}(t)=\omega^{2} \frac{x_{0}-x_{N}}{N}
$$

It is easy to see that the solution of this equation is

$$
L(t)=L(0)+\frac{1}{\alpha}\left(1-e^{-\alpha t}\right) \dot{L}(0)+g_{N}(t)
$$

where

$$
g_{N}(t)=\int_{0}^{t} e^{-\alpha(t-s)} h_{N}(s) d s, \quad h_{N}(s)=\int_{0}^{s} f_{N}\left(s^{\prime}\right) d s^{\prime}
$$

Lemma 4 There exists smooth function $c(t)$ on $R_{+}$such that for any $k \geq 1$ and any $t \geq 0$

$$
\left|x_{k}(t)\right| \leqslant c(t) .
$$

Proof. The solution can be written as in (35)

$$
x_{k}(t)=q_{k}^{(L)}(t)+q_{k}^{(N)}(t) .
$$

We can use formula (35), choosing any contour $\Gamma$, which does not intersect the spectrum of $A$. It is clear that for any $z \in \Gamma$ we have $|\phi(z)|<q<1$ for some $q$. Then

$$
\left|q_{k}^{(L)}(t)\right| \leqslant \frac{|\Gamma|}{2 \pi} \frac{\theta d c+\beta}{1-q} \frac{1}{\omega^{2}} u(t), \quad u(t)=\max _{z \in \Gamma}\left|e^{z t}\right|, \quad c=\max _{z \in \Gamma}|z+\alpha| .
$$

Similarly one can the bound for $q_{k}^{(N)}(t)$.

It follows from this lemma that for any $0<T<\infty$ the sequence $f_{N}(t) \rightarrow 0$, as $N \rightarrow \infty$, uniformly in $t \in[0, T]$. Thus for any $t>0$

$$
\lim _{N \rightarrow \infty} g_{N}(t)=0
$$

and the theorem follows. 


\section{Instability: proofs}

\subsection{Theorem 7}

This is just an exercise to see that the reason for this is the resonance effect: already $x_{1}(t)$ obtains harmonic components with frequencies $\omega, \omega_{0}$, that implies resonance for the particle 2 as its proper frequency is $\omega$. Here

$$
\frac{d^{2} x_{1}(t)}{d t^{2}}+\omega^{2} x_{1}(t)=f(t)=-\frac{1}{\left(\omega_{0} \omega\right)^{2}} \frac{d^{2} z_{0}(t)}{d t^{2}}
$$

with $x_{1}(0)=\dot{x}_{1}(0)=0$. In case $\omega=\omega_{0}$ the proper frequency coincides with the frequency of the external force, and already the first particle will have resonance behaviour that is $\sup _{t \geq 0}\left|x_{1}(t)\right|=\infty$. Consider now the case when $\omega \neq \omega_{0}$. The equation (40) has the solution

$$
x_{1}(t)=\frac{1}{\omega^{2}-\omega_{0}^{2}}\left(\sin \left(\omega_{0} t\right)-\frac{\omega_{0}}{\omega} \sin (\omega t)\right)
$$

That is why already for the particle 2 the resonance occurs. Note however, that the first collision can occur between particles $k$ and $k-1$ for $k>2$.

\subsection{Spectrum, Theorem 8 and Corollary 2}

\section{Remarks concerning spectrum}

Theorem 9 The spectrum $\sigma(A)$ of $A$ is the set

$$
\sigma(A)=\left\{z \in \mathbb{C}:\left|z^{2}+\alpha z+\omega^{2}\right| \leqslant \omega^{2}\right\}
$$

As a corollary we have:

1) if $\alpha \geqslant \sqrt{2} \omega$ then for any $z \in \sigma(A)$ we have $\operatorname{Re}(z) \leqslant 0$ and the equality takes place only if $z=0$.

2) if $\alpha<\sqrt{2} \omega$ then $\sigma(A)$ contains some segment of the imaginary axis together with a neighbourhood.

Proof. Let

$$
R(z)=(A-z E)^{-1}, z \in \mathbb{C}
$$

be the resolvent of $A$. We use the following Lemma.

Lemma 5 Let $\psi=(q, p) \in X, \psi^{\prime}=\left(q^{\prime}, p^{\prime}\right) \in X$ and

$$
R(z) \psi=\psi^{\prime}
$$

Then for any $k=1,2, \ldots$

$$
q_{k}^{\prime}=\sum_{j=0}^{k-1} \phi^{j}(z) l_{k-j}, \quad p_{k}^{\prime}=q_{k}+z q_{k}^{\prime}
$$

where

$$
\phi(z)=\frac{\omega^{2}}{z^{2}+\alpha z+\omega^{2}}, \quad l_{k}(z)=-\frac{(\alpha+z) q_{k}+p_{k}}{z^{2}+\alpha z+\omega^{2}}
$$

Proof. From the definition

$$
\psi=(A-z E) \psi^{\prime} .
$$

Then $q=p^{\prime}-z q^{\prime}$ and $p^{\prime}=q+z q^{\prime}$. Moreover, we have the system of equations

$$
p_{k}=\omega^{2}\left(q_{k-1}^{\prime}-q_{k}^{\prime}\right)-(\alpha+z) p_{k}^{\prime}=\omega^{2}\left(q_{k-1}^{\prime}-q_{k}^{\prime}\right)-(\alpha+z)\left(q_{k}+z q_{k}^{\prime}\right) \text {. }
$$

which, using (43), can be rewritten as

$$
q_{k}^{\prime}=\frac{\omega^{2}}{z^{2}+\alpha z+\omega^{2}} q_{k-1}^{\prime}-\frac{(\alpha+z) q_{k}+p_{k}}{z^{2}+\alpha z+\omega^{2}}=\phi(z) q_{k-1}^{\prime}+l_{k}(z)
$$

Finally

$$
q_{k}^{\prime}=\sum_{j=0}^{k-1} \phi^{j}(z) l_{k-j}
$$


Proof of Theorem 9

Let us consider two cases. Let $|\phi(z)|=q<1$. Note that for any $k$

$$
\left|l_{k}(z)\right| \leqslant\left|\frac{(\alpha+z)+1}{z^{2}+\alpha z+\omega^{2}}\right||| \psi||=c .
$$

It follows that for any $k$

$$
\left|q_{k}^{\prime}\right| \leqslant c \frac{1-q^{k}}{1-q} \leqslant \frac{c}{1-q} .
$$

Then the operator $R(z)$ is bounded and $z \notin \sigma(A)$. Another possibility is $|\phi(z)|>1$. Consider the sequence $\psi_{n}=\left(q^{n}, p^{n}\right) \in X$, where

$$
\begin{aligned}
& q^{n}=0 \\
& p_{k}^{n}= \begin{cases}1, & k<n \\
0, & k \geqslant n .\end{cases}
\end{aligned}
$$

Denote $\psi_{n}^{\prime}=R(z) \psi_{n}, \psi_{n}^{\prime}=\left(\hat{q}^{n}, \hat{p}^{n}\right)$. From formula (44) we get

$$
\hat{q}_{n}^{n}=-\frac{1}{z^{2}+\alpha z+\omega^{2}} \frac{1-\phi^{n}(z)}{1-\phi(z)}
$$

That is why for $|\phi(z)|>1$

$$
\left\|R(z) \psi_{n}\right\| \geqslant \frac{1}{\left|z^{2}+\alpha z+\omega^{2}\right|}\left|\frac{1-\phi^{n}(z)}{1-\phi(z)}\right| \rightarrow \infty, \text { при } n \rightarrow \infty .
$$

However, it is evident that $\left\|\psi_{n}\right\|=1$ and thus $R(z)$ is not bounded. We conclude then that $z \in \sigma(A)$. As the spectrum is a closed subset, we get the proof of the first part of the theorem. Now let us prove the two other assertions.

For $z=a+i b, a, b \in \mathbb{R}$ we have

$$
z^{2}+\alpha z+\omega^{2}=a^{2}-b^{2}+\alpha a+\omega^{2}+i(2 a b+\alpha b) .
$$

Then the inequality $\left|z^{2}+\alpha z+\omega^{2}\right| \leqslant \omega^{2}$ is equivalent to the inequality

$$
\left(a^{2}+\alpha a+\omega^{2}-b^{2}\right)^{2}+b^{2}(2 a+\alpha)^{2} \leqslant \omega^{4} .
$$

or

$$
b^{4}+b^{2}\left((2 a+\alpha)^{2}-2\left(a^{2}+\alpha a+\omega^{2}\right)\right)+\left(a^{2}+\alpha a+\omega^{2}\right)^{2}-\omega^{4} \leqslant 0 .
$$

If we denote

$$
\begin{aligned}
& A=(2 a+\alpha)^{2}-2\left(a^{2}+\alpha a+\omega^{2}\right)=2 a^{2}+2 \alpha a+\alpha^{2}-2 \omega^{2}, \\
& B=\left(a^{2}+\alpha a+\omega^{2}\right)^{2}-\omega^{4}=a(a+\alpha)\left(a^{2}+\alpha a+2 \omega^{2}\right) .
\end{aligned}
$$

the inequality (45) can be rewritten as

$$
h(a, b)=b^{4}+A b^{2}+B \leqslant 0 .
$$

Consider the case $\alpha \geqslant \sqrt{2} \omega$. Then for any $a>0$ and $b \in \mathbb{R}$ we have evidently $h(a, b)>0$, that is $z \notin \sigma(A)$. If $a=0$ then $B=0$ and $A \geqslant 0$. It follows that the unique $b$ such that $h(0, b) \leqslant 0$ is $b=0$. Thus the first assertion is proved.

Let now $\alpha<\sqrt{2} \omega$. For $a=0$ we have $A=\alpha^{2}-2 \omega^{2}<0, B=0$. It follows that there exists $b \in[-\sqrt{-A}, \sqrt{-A}]$ such that $h(0, b) \leqslant 0$. From continuity of $h$ it follows that there is some interval of the imaginary axis around this $b$, belonging to the spectrum.

Remark 4 Stability problems, considered here, cannot be completely treated by spectral methods. In fact, the stability concern the value of $\sup _{t \geqslant 0}\|q(t)\|$. This question was studied by many authors, in particular, by Daletsky and Krein, see ([10]). Their results concern the case of $\epsilon$-dichotomic operators, i.e. when the spectrum of the operator $A$ is the union of two subsets $\sigma_{+}, \sigma_{-}$of the complex plane, such that $\sigma_{+}$belongs to the open right half-plane and $\sigma_{-}$belonds to the open left halfplane. We have shown that for any parameters the spectrum contain the point 0 and thus $A$ is not $\epsilon$-dichotomic operator. 
Proof of Theorem 8 Using formula (34), lemma 5 and the theorem assumptions one gets:

$$
q_{k+1}(t)=-\frac{\epsilon}{2 \pi i} \int_{\Gamma} e^{z t} \phi^{k}(z) d z .
$$

The integrand has two poles (roots of the characteristic equation). Because of $\alpha<\sqrt{2} \omega$ the roots are:

$$
\lambda_{1}=-\frac{\alpha}{2}+i r, \lambda_{2}=-\frac{\alpha}{2}-i r, \quad r=\sqrt{\omega^{2}-\frac{\alpha^{2}}{4}}
$$

By Jordan lemma, for any $a>-\frac{\alpha}{2}$

$$
q_{k+1}(t)=-\frac{\epsilon}{2 \pi i} \int_{a-i \infty}^{a+i \infty} e^{z t} \phi^{k}(z) d z
$$

For $t=\mu k$ we can rewrite this as

$$
\begin{aligned}
& q_{k+1}(\mu k)=F(\mu, k)=-\frac{\epsilon}{2 \pi i} \int_{a-i \infty}^{a+i \infty} \exp \left(k\left(\mu z-\ln \left(z^{2}+\alpha z+\omega^{2}\right)+\ln \omega^{2}\right)\right) d z= \\
& =-\frac{\epsilon}{2 \pi i} \int_{a-i \infty}^{a+i \infty} \exp (k S(z)) d z, \quad S(z)=\mu z-\ln \left(z^{2}+\alpha z+\omega^{2}\right)+\ln \omega^{2} .
\end{aligned}
$$

The mapping $z^{2}+\alpha z+\omega^{2}$ transforms the line $a+i b, b \in \mathbb{R}$ to parabola. Thus one can select holomorphic branch of the logarithm on any such line with $a>-\frac{\alpha}{2}$

$$
\ln \left(z^{2}+\alpha z+\omega^{2}\right)=\ln \left|z^{2}+\alpha z+\omega^{2}\right|+i \arg \left(z^{2}+\alpha z+\omega^{2}\right) .
$$

Now we use the saddle point method. Saddle points are found out from the equation:

$$
S^{\prime}(z)=\mu-\frac{2 z+\alpha}{z^{2}+\alpha z+\omega^{2}}=0
$$

or from the quadratic equation

$$
\mu z^{2}+(\alpha \mu-2) z+\omega^{2} \mu-\alpha=0
$$

The discriminant

$$
D=(\alpha \mu-2)^{2}-4 \mu\left(\omega^{2} \mu-\alpha\right)=\alpha^{2} \mu^{2}+4-4 \mu^{2} \omega^{2}=-4 \mu^{2} \tau^{2}+4 .
$$

and we assume that

$$
\mu>\frac{1}{\tau}
$$

Then $D<0$ and there are two complex saddle points:

$$
z_{ \pm}=z_{ \pm}(\mu)=\frac{2-\alpha \mu \pm i \sqrt{-D}}{2 \mu}=-\frac{\alpha}{2}+\frac{1}{\mu} \pm \frac{i}{\mu} \sqrt{\mu^{2} \tau^{2}-1}
$$

To check that $z_{ \pm}(\mu)$ are simple saddle points, we should find the second derivative

$$
\begin{aligned}
& S^{\prime \prime}\left(z_{ \pm}(\mu)\right)=-\frac{2}{z^{2}+\alpha z+\omega^{2}}+\left.\frac{(2 z+\alpha)^{2}}{\left(z^{2}+\alpha z+\omega^{2}\right)^{2}}\right|_{z=z_{ \pm}(\mu)}=-\frac{2 \mu}{2 z_{ \pm}+\alpha}+\mu^{2}= \\
& =-\frac{\mu}{\frac{1}{\mu} \pm \frac{i}{\mu} \sqrt{\mu^{2} \tau^{2}-1}}+\mu^{2}=\mu^{2}\left(\frac{ \pm i \sqrt{\mu^{2} \tau^{2}-1}}{1 \pm i \sqrt{\mu^{2} \tau^{2}-1}}\right) .
\end{aligned}
$$

This shows that $S^{\prime \prime}\left(z_{ \pm}(\mu)\right) \neq 0$. We shall put $a=a(\mu)=-\frac{\alpha}{2}+\frac{1}{\mu}$ in the formula (477) for $F(\mu, k)$

$$
F(\mu, k)=-\frac{\epsilon}{2 \pi i} \int_{a(\mu)-i \infty}^{a(\mu)+i \infty} \exp (k S(z)) d z .
$$

To use the line $a(\mu)+i b, b \in \mathbb{R}$ as the contour for the saddle point method one should check two following conditions 
1. The function

$$
H(y)=\operatorname{Re}(S(a+i y))
$$

reaches its maximum only at two points: $\operatorname{Im}\left(z_{ \pm}\right)= \pm \frac{1}{\mu} \sqrt{\mu^{2} \tau^{2}-1}$. In fact

$$
\begin{aligned}
& H(y)=\mu a-\frac{1}{2} \ln \left(\left(a^{2}-y^{2}+\alpha a+\omega^{2}\right)^{2}+((2 a+\alpha) y)^{2}\right)+\ln \omega^{2}= \\
& =\mu a+\ln \omega^{2}-\frac{1}{2} \ln h\left(y^{2}\right), \quad h(s)=\left(s-\left(a^{2}+\alpha a+\omega^{2}\right)\right)^{2}+(2 a+\alpha)^{2} s .
\end{aligned}
$$

And it is sufficient to check that the graph of $H(y)$ is the parabola with the vertex:

$$
\begin{aligned}
& s_{0}=-\frac{(2 a+\alpha)^{2}-2\left(a^{2}+\alpha a+\omega^{2}\right)}{2}=-\frac{\frac{4}{\mu^{2}}-2\left(\frac{\alpha^{2}}{4}-\frac{\alpha}{\mu}+\frac{1}{\mu^{2}}-\frac{\alpha^{2}}{2}+\frac{\alpha}{\mu}+\omega^{2}\right)}{2}= \\
& =-\frac{\frac{2}{\mu^{2}}+\frac{\alpha^{2}}{2}-2 \omega^{2}}{2}=\tau^{2}-\frac{1}{\mu^{2}}=\operatorname{Im}\left(z_{ \pm}\right)^{2}
\end{aligned}
$$

2. In some neighbourhoods of the saddle point $z_{ \pm}$the contour goes through two different sectors where $\operatorname{Re}(S(z))<\operatorname{Re}\left(S\left(z_{ \pm}\right)\right)$. To prove this put

$$
\nu=\sqrt{\mu^{2} \tau^{2}-1}
$$

and rewrite the expression for $S^{\prime \prime}\left(z_{ \pm}\right)$:

$$
S^{\prime \prime}\left(z_{ \pm}\right)=\mu^{2} \frac{ \pm i \nu}{1 \pm i \nu}=\mu^{2} \frac{\nu^{2} \pm i \nu}{1+\nu^{2}}=\frac{\nu}{\tau^{2}}(\nu \pm i) .
$$

Further on we agree that the arguments of complex numebrs take values in the interval $(-\pi, \pi]$. Using (49) we obtain:

$$
\arg \left(S^{\prime \prime}\left(z_{+}\right)\right) \in\left(0, \frac{\pi}{2}\right), \quad \arg \left(S^{\prime \prime}\left(z_{-}\right)\right) \in\left(-\frac{\pi}{2}, 0\right) .
$$

The argument of the axis of the saddle point (p. 84 [11]) $z_{ \pm}$is

$$
\phi_{ \pm}=\frac{\pi}{2}-\frac{1}{2} \arg \left(S^{\prime \prime}\left(z_{ \pm}\right)\right) .
$$

It follows that

$$
\phi_{+} \in\left(\frac{\pi}{4}, \frac{\pi}{2}\right), \quad \phi_{-} \in\left(\frac{\pi}{2}, \frac{3 \pi}{4}\right) .
$$

In both cases the angle between the integration contour (i.e. the line $a(\mu)+i b$ ) and the saddle point axis is less then $\frac{\pi}{4}$. That means that the line $a(\mu)+i b$ can be used as the saddle point method contour (see for example,, $\mathrm{p}$. 89 of [11]).

So by formula (5.7.2), p. 88 of [11], we have the asymptotic formula

$$
F(\mu, k) \sim-\frac{\epsilon}{2 \pi i}\left(a_{+} e^{k S\left(z_{+}\right)}+a_{-} e^{k S\left(z_{-}\right)}\right), \text {as } k \rightarrow \infty,
$$

where the constants

$$
a_{ \pm}=\sqrt{\frac{2 \pi}{k\left|S^{\prime \prime}\left(z_{ \pm}\right)\right|}} s_{ \pm}, \quad s_{ \pm}=\exp i \phi_{ \pm} .
$$

We choose the signs of the constants $s_{ \pm}$so that the angle between the saddle point axis (this angle is determined with an accuracy to $\pi$ ) and the contour of integration were acute (see [11]).

One can rewrite the right part of the formula (50)

$$
S\left(z_{ \pm}\right)=\mu z_{ \pm}-\ln \left(\frac{2 z_{ \pm}+\alpha}{\mu}\right)+\ln \omega^{2}=\mu z_{ \pm}-\ln \left(\frac{2}{\mu^{2}}\left(1 \pm i \sqrt{\mu^{2} \tau^{2}-1}\right)\right)+\ln \omega^{2}
$$

Obviously,

$$
\exp \left(k S\left(z_{-}\right)\right)=\overline{\exp \left(k S\left(z_{+}\right)\right)}
$$


and $\arg \left(S^{\prime \prime}\left(z_{-}\right)\right)=-\arg \left(S^{\prime \prime}\left(z_{+}\right)\right)$. Then

$$
\overline{s_{-}}=\exp \left(-i \phi_{-}\right)=\exp \left(-i\left(\frac{\pi}{2}+\arg \left(S^{\prime \prime}\left(z_{+}\right)\right)\right)=\exp (-i \pi) \exp \left(i \phi_{+}\right)=-s_{+} .\right.
$$

Here using (49) we get

$$
\left|S^{\prime \prime}\left(z_{+}\right)\right|=\left|S^{\prime \prime}\left(z_{-}\right)\right|=\frac{\nu}{\tau^{2}} \sqrt{\nu^{2}+1}=\frac{\nu \mu}{\tau} .
$$

and

$$
\begin{gathered}
F(\mu, k) \sim-\frac{\epsilon}{2 \pi i}\left(a_{+} \exp \left(k S\left(z_{+}\right)\right)-\overline{a_{+} \exp \left(k S\left(z_{+}\right)\right)}\right)=-\frac{\epsilon}{\pi} \operatorname{Im}\left(a_{+} \exp \left(k S\left(z_{+}\right)\right)\right. \\
\operatorname{Re}\left(S\left(z_{+}\right)\right)=\mu a(\mu)-\ln \left|\frac{2}{\mu^{2}}\left(1+i \sqrt{\mu^{2} \tau^{2}-1}\right)\right|+\ln \omega^{2}=\mu a(\mu)-\ln \left(\frac{2 \tau}{\mu}\right)+\ln \omega^{2}=f(\mu) \\
\arg \left(1+i \sqrt{\mu^{2} \tau^{2}-1}\right)=\arg S^{\prime \prime}\left(z_{-}\right)+\frac{\pi}{2}=\phi_{+} \\
\operatorname{Im}\left(S\left(z_{+}\right)\right)=\sqrt{\mu^{2} \tau^{2}-1}-\phi_{+}=\nu-\phi_{+}
\end{gathered}
$$

Substituting this to formula (51), we get

$$
F(\mu, k) \sim-\epsilon \sqrt{\frac{2 \tau}{\pi k \nu \mu}} e^{k f(\mu)} \operatorname{Im}\left(e^{i\left(\phi_{+}+k\left(\nu-\phi_{+}\right)\right)}\right)=\epsilon \sqrt{\frac{2 \tau}{\pi k \nu \mu}} e^{k f(\mu)} \sin \left(\Omega(\mu) k+\phi_{0}(\mu)\right)
$$

where

$$
\phi_{0}(\mu)=\phi_{+}=\arctan (\nu), \Omega(\mu)=\nu-\phi_{+}=\nu-\arctan (\nu)
$$

Corollary 2 We need the following

Lemma 6 If $\alpha \leq \sqrt{2} \omega$ there exists $\delta>0$ such that for all $\mu \in\left(\frac{1}{r}, \frac{1}{r}+\delta\right)$

$$
f(\mu)>0 \text {. }
$$

Proof. Using $\tau=\sqrt{\omega^{2}-\frac{\alpha^{2}}{4}}$ and $\alpha<\sqrt{2} \omega$ we get

$$
f\left(\frac{1}{\tau}\right)=-\frac{\alpha}{2 \tau}-\ln \left(\frac{\tau^{2}}{\omega^{2}}\right)+1-\ln 2=1-\ln 2-\frac{x}{\sqrt{1-x^{2}}}-\ln \left(1-x^{2}\right)=h(x), x=\frac{\alpha}{2 \omega}<\frac{1}{\sqrt{2}}
$$

where obviously

$$
h(0)=1-\ln 2>0, \quad h\left(\frac{1}{\sqrt{2}}\right)=0
$$

The following calculation

$$
\begin{aligned}
& h^{\prime}(x)=-\frac{1}{1-x^{2}}-\frac{x^{2}}{\left(1-x^{2}\right) \sqrt{1-x^{2}}}+\frac{2 x}{1-x^{2}}=\frac{-\sqrt{1-x^{2}}-x^{2}+2 x \sqrt{1-x^{2}}}{\left(1-x^{2}\right) \sqrt{1-x^{2}}}= \\
& =-\frac{\left(x-\sqrt{1-x^{2}}\right)^{2}}{\left(1-x^{2}\right) \sqrt{1-x^{2}}}<0, \text { for } x<1 .
\end{aligned}
$$

shows that the function $h$ is monotone decreasing on $[0,1)$. As the function $f$ is smooth, the lemma is proved.

For $t=\mu k, k \rightarrow \infty$ we have:

$$
\begin{aligned}
& R_{k}=r_{k+1}(\mu k)=z_{k}(t)-z_{k+1}(t)=a+q_{k}-q_{k+1} \sim \\
& \sim a+\frac{c}{\sqrt{k-1}} e^{(k-1) f(\mu)}\left(\sin \left(\Omega(\mu)(k-1)+\phi_{0}\right)-\sqrt{\frac{k-1}{k}} e^{f(\mu)} \sin \left(\Omega(\mu) k+\phi_{0}\right)\right)
\end{aligned}
$$

We chose $\mu$ so that $f(\mu)>0$ and $\Omega(\mu)$ is irrational. There exists a subsequence $k_{n}, n=1,2 \ldots$, such that $k_{n} \rightarrow \infty$ as $n \rightarrow \infty$ and

$$
\liminf _{n \rightarrow \infty} \sin \left(\Omega k_{n}+\phi_{0}\right)=-1, \quad \limsup _{n \rightarrow \infty} \sin \left(\Omega k_{n}+\phi_{0}\right)=1
$$

Obviously then:

$$
\liminf _{n \rightarrow \infty} R_{k_{n}}=-\infty, \quad \limsup _{n \rightarrow \infty} R_{k_{n}}=+\infty
$$

Corollary 2 is proved. 


\section{References}

[2] Prigogine I., Herman R. Kinetic theory of vehicular traffic. N.Y.: Elsevier, 1971.

[3] Helbing D. Traffic and related self-driven many particle systems. Rev. Mod. Phys. 73, 1067-1141 (2001).

[4] Feintuch A., Francis B. Infinite chains of kinematic points. Automatica 48 (2012) 901-908.

[5] Qing Hui, Jordan M. Berg. Semistability theory for spatially distributed systems. Proceedings of the IEEE Conference on decision and control, January 2009.

[6] M.R. Jovanovic, B. Bamieh. On the Ill-Posedness of certain vehicular platoon control problems. IEEE transactions on automatic control, Vol.50, NO.9, September 2005.

[7] S.M. Melzer, B.C. Kuo. Optimal regulation of systems described by a countably infinite number of objects. Automatica, Vol. 7, pp. 359-366. Pergamon Press, 1971.

[8] D. Swaroop, J.K. Hedrick. String stability of interconnected systems. IEEE transactions on automatic control, Vol.41, NO.3, March 1996.

[9] Malyshev V.A., Musychka S.A. Dynamical phase transition in the simplest molecular chain model. Theoretical and mathematical physics, 2014, v. 179, No. 1, 123-133.

[10] Daletskij Yu. L., Krejn M. G. Stability of solutions of differential equations in Banach space. 1970. Nauka, Moscow.

[11] N. G. De Bruijn, Asymptotic methods in analysis, Second Edition, 1961. 